PESQUIMAT, Revista de la F.C.M. de la

Universidad Nacional Mayor de San Marcos

Vol. XIII N², pp. 40-58, Lima - Perú, Diciembre 2010

\title{
SOLUCIÓN LOCAL DE UN SISTEMA DE KIRCHHOFF NO LINEAL VISCOELÁSTICO CON TÉRMINO DISIPATIVO
}

\author{
Teófanes Quispe Méndez ${ }^{1}$ \& Luis Enrique Carrillo Díaz ${ }^{2}$
}

Resumen: En el presente trabajo, estudiamos la existencia y unicidad de soluciones locales para el problema mixto relativo a un sistema de ecuaciones de Kirchhoff no lineal viscoelástico con término disipativo.

Palabras clave: Solución Local, Sistema de ecuación de Kirchhoff no lineal viscoelástico, Método de Galerkin, Método del punto fijo.

\section{LOCAL SOLUTION A VISCOELASTIC NONLINEAR KIRCHHOFF'S SYSTEM WITH DISSIPATIVE TERM}

\begin{abstract}
In present work, we study the existence and uniqueness of local solutions for the mixed problem relative to a system of viscoelastic nonlinear Kirchhoff's equation with dissipative term.
\end{abstract}

Key words: Local solution, System of viscoelastic nonlinear Kirchhoff's equation, Galerkin method, Fixed point method.

\footnotetext{
${ }^{1}$ UNMSM, Facultad de Ciencias Matemáticas, e-mail: tquispem@unmsm.edu.pe

${ }^{2}$ UNMSM, Facultad de Ciencias Matemáticas, e-mail: lcarrillod@unmsm.edu.pe
} 


\section{INTRODUCCIÓN}

En este artículo consideramos el problema de valor inicial y de frontera para el siguiente sistema de ecuaciones de Kirchhoff con memoria:

$$
\begin{gathered}
\left.u^{\prime \prime}-M\left(|\nabla u|_{2}^{2}\right) \Delta u+g_{1} * \Delta u-\Delta u^{\prime}=f_{1}(u, v) \text { en } \Omega \times\right] 0, \infty[, \\
\left.v^{\prime \prime}-M\left(|\nabla v|_{2}^{2}\right) \Delta v+g_{2} * \Delta v-\Delta v^{\prime}=f_{2}(u, v) \text { en } \Omega \times\right] 0, \infty[,
\end{gathered}
$$

con condiciones iníciales

$$
\begin{aligned}
& u(x, 0)=u_{0}(x), u^{\prime}(x, 0)=u_{1}(x), \text { en } \Omega, \\
& v(x, 0)=v_{0}(x), v^{\prime}(x, 0)=v_{1}(x), \text { en } \Omega,
\end{aligned}
$$

y condiciones de frontera

$$
\begin{array}{cl}
u(x, t)=, & \text { en } \partial \Omega \times] 0, \infty[, \\
v(x, t)=0, & \text { en } \partial \Omega \times] 0, \infty[,
\end{array} .
$$

donde $\Omega$ es un conjunto abierto y acotado de $\mathbb{R}^{n}$ con frontera suficientemente regular $\partial \Omega, \nabla$ es el operador gradiente, $\Delta$ es el operador laplaciano, $M(s)$ es una función real positiva de clase $C^{1}$ para $s \geq 0, g_{i}(t), i=1,2$, son funciones reales no negativas de clase $C^{1}$ para $t \geq 0, f_{i}(s, r)$, $i=1,2$, son funciones reales no lineales continuamentc diferenciables para $(s, r) \in \mathbb{R}^{2}, w^{\prime}:=\frac{\partial w}{\partial t}$, $w^{\prime \prime}:=\frac{\partial^{2} w}{\partial t^{2}},|\nabla w|_{2}^{2}:=\int_{\Omega}|\nabla w(x, t)|^{2} d x$ y $(g * w)(x, t):=\int_{0}^{t} g(t-s) w(x, s) d s$.

El caso $n=1$ y $u=v$, la ecuación (1.1) describe vibraciones transversales no lineales de una cuerda de material viscoelástico, fuertemente tensa entre dos puntos fijos $x=0$ y $x=L$, en el eje $x$ del plano $x u$. En estas condiciones la ecuación resultante es

$$
\rho h u^{\prime \prime}-\left(p_{o}+\frac{E h}{2 L}\left|\frac{\partial u}{\partial x}\right|_{2}^{2}\right) \frac{\partial^{2} u}{\partial x^{2}}+g * \frac{\partial^{2} u}{\partial x^{2}}-\beta \frac{\partial^{2} u^{\prime}}{\partial x^{2}}=f(u),
$$

donde $u=u(x, t)$ es el desplazamiento transversal en el espacio de coordenada $x$ y en el tiempo $t$, $\rho$ es la densidad de masa, $h$ es el área de la sección transversal de la cuerda, $p_{0}$ es la tensión inicial, $E$ es el módulo de Young del material, $\beta$ es el coeficiente de la fuerza amortiguadora, $g(t)$ es la función de relajación, y $f(u)$ es la fuerza restauradora. Cuando en (1.7), $\beta \equiv g \equiv 0$ y la cuerda es de material elástico se tiene que la ecuación (1.7) es la propuesta y estudiada por Kirchhoff en [4]. El caso general $n \geq 1$ y $u=v$, la ecuación (1.1) tiene diversas aplicaciones, como en el área de la óptica no lineal, física del plasma y mecánica de fluidos, entre otras.

El caso $M \equiv 1, g_{i} \equiv 0$ y sin término disipativo, el sistema (1.1) - (1.2) describe la interacción de ciertas partículas elementales en un campo electromagnético, llamadas mesones. Un modelo matemático clásico que describe la interacción de los mesones es el sistema propuesto por Segal [11] que mostramos a continuación:

$$
\begin{aligned}
& u^{\prime \prime}-\Delta u+\alpha^{2} u+g^{2} v^{2} u=0 \\
& v^{\prime \prime}-\Delta v+\beta^{2} v+h^{2} u^{2} v=0
\end{aligned}
$$

donde $\alpha$ y $\beta$ son las masas de los mesones $u$ y $v$ respectivamente y $g, h$ son las constantes de interacción.

Cuando $g_{i}$ son funciones no triviales, $M \equiv 1$ y sin considerar término disipativo, el sistema (1.1) - (1.2) fue investigado por Andrade y Mognon [1], quienes obtienen existencia global para 
$f_{1}(u, v)=-|u|^{\rho-1} u|v|^{\rho}$ y $f_{2}(u, v)=-|v|^{\rho-1} v|u|^{\rho}$; por su parte Santos [10], obtiene existencia global y decaimiento exponencial para $f_{1}(u, v)=-\alpha(u-v)$ y $f_{2}(u, v)=\alpha(u-v)$. Cuando $g_{i} \equiv 0, M \equiv 1$ y $\sin$ término disipativo, el sistema (1.1) - (1.2) fue también estudiado por Milla Miranda y Medeiros [6], quienes obtienen existencia global para $f_{1}(u, v)=|v|^{\rho+2}|u|^{\rho} u-u$ y $f_{2}(u, v)=|u|^{\rho+2}|v|^{\rho} v-v$; asimismo Li and Tsai [5], obtienen existencia local, existencia global y singularidad de soluciones para $f_{1}(u, v)=-m_{1}^{2} u-4 \lambda(u+\alpha v)^{3}-2 \beta u v^{2}$ y $f_{2}(u, v)=$ $-m_{2}^{2} v-4 \alpha \lambda(u+\alpha v)^{3}-2 \beta u^{2} v$. Cuando $g_{i} \equiv 0$ y $M$ es una función no trivial, el sistema (1.1) - (1.2) fue estudiado por Quispe Méndez [7, 8], quien obtiene soluciones locales y singularidad de soluciones para $f_{i}$ específicas y sin término disipativo; con relación a análogo sistema, Wu y Tsai [14], obtienen existencia local y singularidad de soluciones para $f_{i}$ genérica y adicionando término disipativo.

En este trabajo probaremos la existencia y unicidad de soluciones locales del problema (1.1) - (1.6), cuando $g_{i}$ son funciones continuas no negativas, $M$ es una función continua positiva y las $f_{i}$ son funciones reales no lineales. Primero probaremos la existencia y unicidad de solución global de un problema lineal asociado a $(1.1)-(1.6)$ en el caso $u=v$, utilizando el método de Galerkin. Asimismo, obtendremos una estimativa para las correspondientes soluciones. En segundo lugar, linealizaremos el problema (1.1) - (1.6) para elementos de un espacio $G_{T_{0}, R_{0}}$, llamado espacio de soluciones, luego obtendremos sus soluciones en $G_{T_{0}, R_{0}}$ y después, por argumentos del teorema de punto fijo de Banach, se obtendran las soluciones locales del problema (1.1) - (1.6) sobre un intervalo $\left[0, T_{0}\right]$, donde $T_{0}>0$ depende de los datos iníciales y de los parámetros del problema. La unicidad del par de soluciones será obtenida utilizando las estimativas de las soluciones del problema lineal. En la discusión del problema emplearemos las estrategias y herramientas inspiradas en los trabajos de Andrade y Mognon [1], Wu y Tsai [14] y Quispe Méndez [9].

\section{PRELIMINARES}

En esta sección presentamos algunas notaciones, conceptos y resultados sin demostración, los cuales serán usados en el desarrollo del presente trabajo.

Sea $\Omega$ un conjunto abierto y acotado de $\mathbb{R}^{n}$ con frontera suficientemente regular $\partial \Omega$. Denotamos el producto interno y la norma de $L^{2}(\Omega)$ y $L^{p}(\Omega)$, con $(.,$.$) y |\cdot|_{p}$, respectivamente, para $1 \leq p \leq \infty$. Además $((.,)$.$) y \|$.$\| , denotaran el producto interno y la norma de H_{0}^{1}(\Omega)$, donde $((u, v)):=\int_{\Omega} \nabla u(x) . \nabla v(x) d x$ es la forma de Dirichlet.

Sea $X$ un espacio de Banach, $T$ y $p$ números reales tales que $0<T \leq \infty$ y $1 \leq p \leq \infty$. Representamos con $L^{p}(0, T ; X)$ al espacio de Banach de las funciones vectoriales $\left.u:\right] 0, T[\longrightarrow$ $X$ medibles con $\|u(t)\|_{X} \in L^{p}(0, T)$, dotado de la norma

$$
\begin{gathered}
\|u\|_{L^{p}(0, T ; X)}:=\left(\int_{0}^{T}\|u(t)\|_{X}^{p} d t\right)^{\frac{1}{p}}, 1 \leq p<\infty, \\
\|u\|_{L^{\infty}(0, T ; X)}:=\sup _{0<t<T} \operatorname{ess}\|u(t)\|_{X}, p=\infty .
\end{gathered}
$$

Similarmente, cuando $0<T<\infty$, representamos con $C([0, T] ; X)$ al espacio de Banach de las funciones continuas $u:[0, T] \longrightarrow X$, dotado de la norma

$$
\|u\|_{C([0, T] ; X)}:=\sup _{0 \leq t \leq T}\|u(t)\|_{X} .
$$

Denotamos $w^{\prime}:=\frac{\partial w}{\partial t}, w^{\prime \prime}:=\frac{\partial^{2} w}{\partial t^{2}}, w(t)(x):=w(x, t), L^{1}:=L^{1}(0, T)$ y $L^{\infty}:=L^{\infty}(\Omega)$. 
Hipótesis. Imponemos sobre las funciones reales $\mu(t), g_{i}(t), M(s)$ y $f_{i}(r, s)$ algunas condiciones, las cuales en ciertas situaciones son propias del problema que modelan y en otras son de naturaleza técnica para el éxito del método empleado.

(H1) $\mu \in C\left(\left[0, \infty[), \mu^{\prime} \in L^{2}(0, \infty)\right.\right.$ y $\mu(t) \geq m_{0}>0, \forall t \geq 0$, donde $m_{0}$ es una constante positiva.

(H2) $g_{i} \in C^{1}\left(\left[0, \infty[)\right.\right.$, acotada, $g_{i}(t) \geq 0, \forall t \geq 0$,

$$
m_{0}-\int_{0}^{\infty} g_{i}(s) d s:=l_{i}>0
$$

para $i=0,1,2$, donde $m_{0}$ es la constante dada en ( $\left.H 1\right)$, además suponemos que existen constantes positivas $C_{1}$ y $C_{2}$ tales que

$$
-C_{1} g_{i}(t) \leq g_{i}^{\prime}(t) \leq-C_{2} g_{i}(t), \forall t \geq 0
$$

para $i=0,1,2$.

(H3) $M \in C^{1}\left(\left[0, \infty[)\right.\right.$ y $M(s) \geq m_{0}>0, \forall s \geq 0$, donde $m_{0}$ es una constante dada en $(H 1)$.

(H4) $f_{i}(0,0)=0, i=1,2$ y existe una constante positiva $K$ tal que

$$
\begin{aligned}
\left|f_{i}\left(r_{1}, s_{1}\right)-f_{i}\left(r_{2}, s_{2}\right)\right| \leq & K\left[\left(\left|r_{1}\right|^{\alpha}+\left|r_{2}\right|^{\alpha}\right)\left|r_{1}-r_{2}\right|\right. \\
& \left.+\left(\left|s_{1}\right|^{\beta}+\left|s_{2}\right|^{\beta}\right)\left|s_{1}-s_{2}\right|\right],
\end{aligned}
$$

para cada $\left(r_{1}, s_{1}\right),\left(r_{2}, s_{2}\right) \in \mathbb{R}^{2}, i=1,2$, con $0 \leq \alpha, \beta \leq \frac{2}{n-2}$ para $n \geq 3$ ó $\alpha, \beta \geq 0$ para $n \leq 2$.

Lema 2.1 (Desigualdad de Sobolev-Poincaré [2]). Si $2 \leq p \leq \frac{2 n}{n-2}$ para $n \geq 3$ ó $p \geq 2$ para $n \leq 2$, entonces existe una constante positiva $B_{1}$ tal que

$$
|u|_{p} \leq B_{1}\|u\|, \forall u \in H_{0}^{1}(\Omega)
$$

$y$

$$
\|u\| \leq B_{1}|\Delta u|_{2}, \forall u \in H_{0}^{1}(\Omega) \cap H^{2}(\Omega) .
$$

Lema 2.2 (Desigualdad de Young [2]). Sea $u \in L^{p}\left(\mathbb{R}^{m}\right)$ y $v \in L^{q}\left(\mathbb{R}^{m}\right)$ con $1 \leq p \leq \infty$, $1 \leq q \leq \infty$ y $r$ un número real verificando $\frac{1}{r}=\frac{1}{p}+\frac{1}{q}-1 \geq 0$. Entonces $u * v \in L^{r}\left(\mathbb{R}^{m}\right)$ y

$$
\|u * v\|_{L^{r}\left(\mathbb{R}^{m}\right)} \leq\|u\|_{L^{p}\left(\mathbb{R}^{m}\right)}\|v\|_{L^{q}\left(\mathbb{R}^{m}\right)},
$$

donde la convolución de u con v está definida por

$$
(u * v)(x):=\int_{\mathbb{R}^{m}} u(x-y) v(y) d y .
$$

Lema 2.3. Si $g \in C^{1}\left(\left[0, \infty[)\right.\right.$ y $w \in C^{1}\left([0, T] ; L^{2}(\Omega)\right)$, entonces

$$
\begin{aligned}
\int_{0}^{t} g(t-s)\left(w(s), w^{\prime}(t)\right) d s= & -\frac{1}{2} \frac{d}{d t}\left[(g \square w)(t)-\left(\int_{0}^{t} g(s) d s\right)|w(t)|_{2}^{2}\right] \\
& -\frac{1}{2} g(t)|w(t)|_{2}^{2}+\frac{1}{2}\left(g^{\prime} \square w\right)(t),
\end{aligned}
$$


donde

$$
(v \square w)(t):=\int_{0}^{t} v(t-s)|w(t)-w(s)|_{2}^{2} d s .
$$

Demostración. Diferenciando el término $g \square w$, se obtiene el resultado.

Lema 2.4 (Desigualdad de Gronwall [15]). Sean $f, g:[a, b] \rightarrow \mathbb{R}$ funciones continuas, con $g$ no decreciente y $h \in L^{1}(a, b)$, las cuales satisfacen la desigualdad

$$
f(t) \leq g(t)+\int_{a}^{t} h(s) f(s) d s, \forall t \in[a, b] .
$$

Entonces,

$$
f(t) \leq g(t) \exp \left(\int_{a}^{t} h(s) d s\right), \forall t \in[a, b] .
$$

Definición 2.5. Al par de funciones $(u, v)$ se le llamada solución del problema (1.1) - (1.6) sobre $[0, T]$, si las funciones $u, v: \Omega \times[0, T] \rightarrow \mathbb{R}$ satisfacen las condiciones (1.3) - (1.6) y se verifican las igualdades siguientes:

$$
\begin{aligned}
& u^{\prime \prime}-M\left(|\nabla u|_{2}^{2}\right) \Delta u+g_{1} * \Delta u-\Delta u^{\prime}=f_{1}(u, v) \text { en } L^{2}\left(0, T ; H^{-1}(\Omega)\right), \\
& v^{\prime \prime}-M\left(|\nabla v|_{2}^{2}\right) \Delta v+g_{2} * \Delta v-\Delta v^{\prime}=f_{2}(u, v) \text { en } L^{2}\left(0, T ; H^{-1}(\Omega)\right) .
\end{aligned}
$$

\section{PROBLEMA LINEAL}

En esta sección estudiaremos la solución global del siguiente problema lineal mixto:

$$
\begin{array}{ll}
u^{\prime \prime}-\mu(t) \Delta u+g_{0} * \Delta u-\Delta u^{\prime}=h(x, t) & \text { en } \Omega \times] 0, T[, \\
u=0 & \text { en } \partial \Omega \times] 0, T[, \\
u(x, 0)=u_{0}(x), u^{\prime}(x, 0)=u_{1}(x) & \text { en } \Omega,
\end{array}
$$

donde $T$ es un número real positivo fijo, escogido arbitrariamente.

Teorema 3.1. Supongamos que las funciones $\mu$ y $g_{0}$ satisfacen las hipótesis (H1) y (H2), respectivamente, $u_{0} \in H_{0}^{1}(\Omega) \cap H^{2}(\Omega), u_{1} \in L^{2}(\Omega)$ y $h \in L^{2}\left(0, T ; L^{2}(\Omega)\right)$. Entonces el problema (3.1) admite solución única u sobre $[0, T]$ tal que

$$
\begin{aligned}
& u \in C\left([0, T] ; H_{0}^{1}(\Omega) \cap H^{2}(\Omega)\right), \\
& u^{\prime} \in C\left([0, T] ; L^{2}(\Omega)\right) \cap L^{2}\left(0, T ; H_{0}^{1}(\Omega)\right), \\
& u^{\prime \prime} \in L^{2}\left(0, T ; L^{2}(\Omega)\right) .
\end{aligned}
$$

Además, la solución u verifica la estimativa

$$
E(t) \leq\left[E_{0}^{\frac{1}{2}}+2 \int_{0}^{t}|h(s)|_{2} d s\right]^{2} \exp \left(\int_{0}^{t} \frac{\left|\mu^{\prime}(s)\right|}{\mu_{0}(s)} d s\right), \forall t \in[0, T],
$$

donde

$$
\begin{aligned}
& E(t):=\frac{1}{10}\left|u^{\prime}(t)\right|_{2}^{2}+\frac{1}{10}|\Delta u(t)|_{2}^{2}+\frac{1}{2} \mu_{0}(t)\|u(t)\|^{2}, \\
& \mu_{0}(t):=\mu(t)-\int_{0}^{t} g_{0}(s) d s, \\
& E_{0}:=\frac{9}{10}\left|u_{1}\right|_{2}^{2}+\frac{3}{10}\left|\Delta u_{0}\right|_{2}^{2}+\frac{1}{2} \mu(0)\left\|u_{0}\right\|^{2} .
\end{aligned}
$$


Demostración. Procedemos en seis etapas.

Soluciones Aproximadas. Sean $\left\{\omega_{k}\right\}_{k \in \mathbb{N}}$ una base en $H_{0}^{1}(\Omega) \cap H^{2}(\Omega)$ y $V_{m}=\left[\omega_{1}, \omega_{2}, \ldots, \omega_{m}\right]$ el subespacio generado por los primeros $m$ vectores $\omega_{1}, \omega_{2}, \ldots, \omega_{m}$ de $\left\{\omega_{k}\right\}_{k \in \mathbb{N}}$. Consideremos

$$
u_{m}(t)=\sum_{j=1}^{m} r_{j m}(t) \omega_{j},
$$

las soluciones aproximadas en $V_{m}$ del problema (3.1), donde las funciones $r_{j m}(t), j=1,2, \ldots, m$, son determinadas del siguiente problema en ecuaciones diferenciales ordinarias, para $w \in V_{m}$

$$
\begin{aligned}
& \left(u_{m}^{\prime \prime}(t), w\right)+\mu(t)\left(\left(u_{m}(t), w\right)\right) \\
& \quad-\int_{0}^{t} g_{0}(t-s)\left(\left(u_{m}(s), w\right)\right) d s+\left(\left(u_{m}^{\prime}, w\right)\right)=(h(t), w), \\
& u_{m}(0)=u_{0 m}, \quad u_{m}^{\prime}(0)=u_{1 m},
\end{aligned}
$$

donde

$$
\begin{aligned}
& u_{0 m}=\sum_{j=1}^{m} r_{0 j m} w_{j}, \quad u_{0 m} \rightarrow u_{0} \quad \text { fuerte en } H_{0}^{1}(\Omega) \cap H^{2}(\Omega), \\
& u_{1 m}=\sum_{j=1}^{m} r_{1 j m} w_{j}, \quad u_{1 m} \rightarrow u_{1} \quad \text { fuerte en } L^{2}(\Omega) .
\end{aligned}
$$

El Teorema de Carathéodory [3], nos garantiza la existencia de una solución local $u_{m}$ del problema aproximado (3.3) en el intervalo $\left[0, T_{m}[\right.$. Las siguientes estimativas a priori nos permitirán extender la $u_{m}$ al intervalo $[0, T]$, con $T$ independiente de $m$.

Estimativa I. Considerando $w=u_{m}^{\prime}(t)$ en (3.3), obtenemos

$$
\begin{aligned}
& \frac{1}{2} \frac{d}{d t}\left|u_{m}^{\prime}(t)\right|_{2}^{2}+\frac{1}{2} \mu(t) \frac{d}{d t}\left\|u_{m}(t)\right\|^{2} \\
& \quad-\int_{0}^{t} g_{0}(t-s)\left(\left(u_{m}(s), u_{m}^{\prime}(t)\right)\right) d s+\left\|u_{m}^{\prime}(t)\right\|^{2}=\left(h(t), u_{m}^{\prime}(t)\right) .
\end{aligned}
$$

Del Lema 2.3, resulta

$$
\begin{array}{r}
-\int_{0}^{t} g_{0}(t-s)\left(\left(u_{m}(s), u_{m}^{\prime}(t)\right)\right) d s=\frac{1}{2} \frac{d}{d t}\left[\left(g_{0} \square \nabla u_{m}\right)(t)\right. \\
\left.\left.-\left(\int_{0}^{t} g_{0}(s) d s\right) \| u_{m}(t)\right) \|^{2}\right] \\
\left.+\frac{1}{2} g_{0}(t) \| u_{m}(t)\right) \|^{2} \\
-\frac{1}{2}\left(g_{0}^{\prime} \square \nabla u_{m}\right)(t) .
\end{array}
$$

De (3.5) y (3.6), se obtiene

$$
\begin{array}{r}
\frac{d}{d t} E_{1}(t)=\left(h(t), u_{m}^{\prime}(t)\right)+\frac{1}{2} \mu^{\prime}(t)\left\|u_{m}(t)\right\|^{2} \\
\left.-\frac{1}{2} g_{0}(t) \| u_{m}(t)\right) \|^{2}+\frac{1}{2}\left(g_{0}^{\prime} \square \nabla u_{m}\right)(t),
\end{array}
$$


donde

$$
\begin{aligned}
& E_{1}(t):=\frac{1}{2}\left|u_{m}^{\prime}(t)\right|_{2}^{2}+\frac{1}{2} \mu_{0}(t)\left\|u_{m}(t)\right\|^{2} \\
+ & \frac{1}{2}\left(g_{0} \square \nabla u_{m}\right)(t)+\int_{0}^{t}\left\|u_{m}^{\prime}(s)\right\|^{2} d s, \\
\mu_{0}(t):= & \mu(t)-\int_{0}^{t} g_{0}(s) d s .
\end{aligned}
$$

De (3.7), se tiene

$$
\frac{d}{d t} E_{1}(t) \leq|h(t)|_{2}\left|u_{m}^{\prime}(t)\right|_{2}+\frac{1}{2}\left|\mu^{\prime}(t)\right|\left\|u_{m}(t)\right\|^{2} .
$$

De (3.9), se obtiene

$$
\frac{d}{d t} E_{1}^{\frac{1}{2}}(t) \leq|h(t)|_{2}+\frac{1}{2}\left[\frac{\left|\mu^{\prime}(t)\right|}{\mu_{0}(t)}\right] E_{1}^{\frac{1}{2}}(t)
$$

Luego de integrar (3.10) y de hacer uso de la Desigualdad de Gronwall, se obtiene

$$
E_{1}(t) \leq\left[E_{1}^{\frac{1}{2}}(0)+\int_{0}^{t}|h(s)|_{2} d s\right]^{2} \exp \left(\int_{0}^{t} \frac{\left|\mu^{\prime}(s)\right|}{\mu_{0}(s)} d s\right),
$$

donde

De (3.11), se tiene

$$
E_{1}(0):=\frac{1}{2}\left|u_{1 m}\right|_{2}^{2}+\frac{1}{2} \mu(0)\left\|u_{0 m}\right\|^{2}
$$

$$
\left|u_{m}^{\prime}(t)\right|_{2}^{2}+\left\|u_{m}(t)\right\|^{2}+\int_{0}^{t}\left\|u_{m}^{\prime}(s)\right\|^{2} d s \leq K_{1}, \forall t \in[0, T],
$$

donde $K_{1}$ es una constante positiva independiente de $m$.

Estimativa II. Tomando $w=u_{m}^{\prime \prime}(t)$ en (3.3), obtenemos

$$
\begin{aligned}
& \left|u_{m}^{\prime \prime}(t)\right|_{2}^{2}+\mu(t) \frac{d}{d t}\left(\left(u_{m}(t), u_{m}^{\prime}(t)\right)\right)-\mu(t)\left\|u_{m}^{\prime}(t)\right\|^{2} \\
& -\int_{0}^{t} g_{0}(t-s)\left(\left(u_{m}(s), u_{m}^{\prime \prime}(t)\right)\right) d s+\frac{1}{2} \frac{d}{d t}\left\|u_{m}^{\prime}(t)\right\|^{2}=\left(h(t), u_{m}^{\prime \prime}(t)\right) .
\end{aligned}
$$

Diferenciando la integal $\int_{0}^{t} g_{0}(t-s)\left(\left(u_{m}(s), u_{m}^{\prime}(t)\right)\right) d s$, se obtiene

$$
\begin{aligned}
&-\int_{0}^{t} g_{0}(t-s)\left(\left(u_{m}(s), u_{m}^{\prime \prime}(t)\right)\right) d s=-\frac{d}{d t} {\left[\int_{0}^{t} g_{0}(t-s)\left(\left(u_{m}(s), u_{m}^{\prime}(t)\right)\right) d s\right] } \\
&+\int_{0}^{t} g_{0}^{\prime}(t-s)\left(\left(u_{m}(s), u_{m}^{\prime}(t)\right)\right) d s \\
&+g_{0}(0)\left(\left(u_{m}(t), u_{m}^{\prime}(t)\right)\right) .
\end{aligned}
$$

De (3.13) y (3.14) obtenemos

$$
\begin{aligned}
\left|u_{m}^{\prime \prime}(t)\right|_{2}^{2} & +\frac{d}{d t}\left[\frac{1}{2}\left\|u_{m}^{\prime}(t)\right\|^{2}+\mu(t)\left(\left(u_{m}(t), u_{m}^{\prime}(t)\right)\right)\right. \\
-\int_{0}^{t} g_{0}(t-s) & \left.\left(\left(u_{m}(s), u_{m}^{\prime}(t)\right)\right) d s\right]=\left(h(t), u_{m}^{\prime \prime}(t)\right) \\
& +\mu^{\prime}(t)\left(\left(u_{m}(t), u_{m}^{\prime}(t)\right)\right)+\mu(t)\left\|u_{m}^{\prime}(t)\right\|^{2} \\
& -g_{0}(0)\left(\left(u_{m}(t), u_{m}^{\prime}(t)\right)\right)-\int_{0}^{t} g_{0}^{\prime}(t-s)\left(\left(u_{m}(s), u_{m}^{\prime}(t)\right)\right) d s .
\end{aligned}
$$


Por (H2), utilizando la desigualdad $a b \leq \delta a^{2}+\frac{1}{4 \delta} b^{2}, \forall a, b \geq 0, \forall \delta>0$ y la Desigualdad de Hölder, obtenemos

$$
\begin{gathered}
-\int_{0}^{t} g_{0}^{\prime}(t-s)\left(\left(u_{m}(s), u_{m}^{\prime}(t)\right)\right) d s \leq \eta\left\|u_{m}^{\prime}(t)\right\|^{2} \\
+\frac{C_{1}^{2}}{4 \eta}\left|g_{0}\right|_{L^{1}}\left(g_{0} *\left\|u_{m}\right\|^{2}\right)(t), \\
-g_{0}(0)\left(\left(u_{m}(t), u_{m}^{\prime}(t)\right)\right) \leq \eta\left\|u_{m}^{\prime}(t)\right\|^{2}+\frac{g_{0}^{2}(0)}{4 \eta}\left\|u_{m}(t)\right\|^{2}, \\
\mu^{\prime}(t)\left(\left(u_{m}(t), u_{m}^{\prime}(t)\right)\right) \leq \eta\left\|u_{m}^{\prime}(t)\right\|^{2}+\frac{1}{4 \eta}\left|\mu^{\prime}(t)\right|^{2}\left\|u_{m}(t)\right\|^{2}, \\
\left(h(t), u_{m}^{\prime \prime}(t)\right) \leq \varepsilon\left|u_{m}^{\prime \prime}(t)\right|_{2}^{2}+\frac{1}{4 \varepsilon}|h(t)|_{2}^{2}, \\
-\mu(t)\left(\left(u_{m}(t), u_{m}^{\prime}(t)\right)\right) \leq \eta\left\|u_{m}^{\prime}(t)\right\|^{2}+\frac{1}{4 \eta}|\mu(t)|^{2}\left\|u_{m}(t)\right\|^{2}
\end{gathered}
$$

y

$$
\begin{array}{r}
\int_{0}^{t} g_{0}(t-s)\left(\left(u_{m}(s), u_{m}^{\prime}(t)\right)\right) d s \leq \eta\left\|u_{m}^{\prime}(t)\right\|^{2} \\
+\frac{1}{4 \eta}\left|g_{0}\right|_{L^{1}}\left(g_{0} *\left\|u_{m}\right\|^{2}\right)(t),
\end{array}
$$

donde $0<\varepsilon<1$ y $0<\eta<\frac{1}{4}$ son constantes. Usando la Desigualdad de Young para la convolución, se tiene

$$
\int_{0}^{t}\left(g_{0} *\left\|u_{m}\right\|^{2}\right)(s) d s \leq\left(\int_{0}^{t} g_{0}(s) d s\right) \int_{0}^{t}\left\|u_{m}(s)\right\|^{2} d s .
$$

Integrando (3.15), usando (3.16) - (3.19), y después (3.20) - (3.22), resulta

$$
\begin{aligned}
(1-\varepsilon) \int_{0}^{t}\left|u_{m}^{\prime \prime}(s)\right|_{2}^{2} d s & +\left(\frac{1}{2}-2 \eta\right)\left\|u_{m}^{\prime}(t)\right\|^{2} \\
\leq & \frac{1}{2}\left\|u_{1 m}\right\|^{2}+\mu(0)\left\|u_{0 m}\right\|\left\|u_{1 m}\right\| \\
& +\int_{0}^{t}[3 \eta+\mu(s)]\left\|u_{m}^{\prime}(s)\right\|^{2} d s \\
& +\frac{1}{4 \eta} \int_{0}^{t}\left[g_{0}^{2}(0)+\left|\mu^{\prime}(s)\right|^{2}+C_{1}^{2}\left|g_{0}\right|_{L^{1}}^{2}\right. \\
& \left.+\left|g_{0}\right|_{L^{1}}\left|g_{0}\right|_{L^{\infty}}\right]\left\|u_{m}(s)\right\|^{2} d s \\
& +\frac{1}{4 \varepsilon} \int_{0}^{t}|h(s)|_{2}^{2} d s+\frac{1}{4 \eta}|\mu(t)|^{2}\left\|u_{m}(t)\right\|^{2} .
\end{aligned}
$$

De (3.23), escogiendo $\varepsilon=\frac{3}{4}$ y $\eta=\frac{1}{8}$, y utilizando la estimativa (3.12), se tiene

$$
\begin{aligned}
\frac{1}{4} \int_{0}^{t}\left|u_{m}^{\prime \prime}(s)\right|_{2}^{2} d s+ & \frac{1}{4}\left\|u_{m}^{\prime}(t)\right\|^{2} \\
\leq & \frac{1}{2}\left\|u_{1 m}\right\|^{2}+\mu(0)\left\|u_{0 m}\right\|\left\|u_{1 m}\right\| \\
& +\frac{1}{3}\|h\|_{L^{2}\left(0, T ; L^{2}(\Omega)\right)}^{2}+\frac{1}{2} \mu_{0}^{2} K_{1} \\
& +2\left(g^{2}(0)+\mu_{1}^{2}+C_{1}^{2}|g|_{L^{1}}^{2}+|g|_{L^{1}}|g|_{L^{\infty}}\right) K_{1} T \\
& +\left(\frac{3}{8}+\mu_{0}\right) \int_{0}^{t}\left\|u_{m}^{\prime}(s)\right\|^{2} d s,
\end{aligned}
$$


donde $\mu_{0}:=\sup _{t \in[0, T]}|\mu(t)|$ y $\mu_{1}:=\sup _{t \in[0, T]}\left|\mu^{\prime}(t)\right|$.

De (3.24) por la Desigualdad de Gronwall, tenemos

$$
\left\|u_{m}^{\prime}(t)\right\|^{2}+\int_{0}^{t}\left|u_{m}^{\prime \prime}(s)\right|_{2}^{2} d s \leq K_{2}, \forall t \in[0, T]
$$

donde $K_{2}$ es una constante positiva independiente de $m$.

Estimativa III. Tomando $w=-\Delta u_{m}(t)$ en (3.3), obtenemos

$$
\begin{aligned}
\frac{d}{d t}\left[\frac{1}{2}\left|\Delta u_{m}(t)\right|_{2}^{2}-\right. & \left.\left(u_{m}^{\prime}(t), \Delta u_{m}(t)\right)\right]+\mu(t)\left|\Delta u_{m}(t)\right|_{2}^{2} \\
= & \left(h(t),-\Delta u_{m}(t)\right)+\left\|u_{m}^{\prime}(t)\right\|^{2} \\
& +\int_{0}^{t} g_{0}(t-s)\left(\Delta u_{m}(s), \Delta u_{m}(t)\right) d s .
\end{aligned}
$$

Por la Desigualdad de Hölder, resulta

$$
\begin{array}{r}
\int_{0}^{t} g_{0}(t-s)\left(\Delta u_{m}(s), \Delta u_{m}(t)\right) d s \leq \eta\left|\Delta \dot{u_{m}}(t)\right|_{2}^{2} \\
+\frac{1}{4 \eta}\left|g_{0}\right|_{L^{1}}\left(g_{0} *\left|\Delta u_{m}\right|_{2}^{2}\right)(t),
\end{array}
$$

donde $0<\eta<\frac{1}{2} m_{0}$ constante. De (3.26) y por (3.27), se tiene

$$
\begin{gathered}
\frac{d}{d t}\left[\frac{1}{2}\left|\Delta u_{m}(t)\right|_{2}^{2}-\left(u_{m}^{\prime}(t), \Delta u_{m}(t)\right)\right]+(\mu(t)-\eta)\left|\Delta u_{m}(t)\right|_{2}^{2} \\
\leq|h(t)|_{2}\left|\Delta u_{m}(t)\right|_{2}+\left\|u_{m}^{\prime}(t)\right\|^{2} \\
+\frac{1}{4 \eta}\left|g_{0}\right|_{L^{1}}\left(g_{0} *\left|\Delta u_{m}\right|_{2}^{2}\right)(t) .
\end{gathered}
$$

Aplicando Desigualdad de Young para la convolución y utilizando la desigualdad $a b \leq \delta a^{2}+\frac{1}{4 \delta} b^{2}$, $\forall a, b \geq 0, \forall \delta>0$, se obtienen

$$
\begin{aligned}
\int_{0}^{t}\left(g_{0} *\left|\Delta u_{m}\right|_{2}^{2}\right)(s) d s & \leq\left(\int_{0}^{t} g_{0}(s) d s\right) \int_{0}^{t}\left|\Delta u_{m}(s)\right|_{2}^{2} d s \\
\left(u_{m}^{\prime}(t), \Delta u_{m}(t)\right) d s & \leq \lambda\left|\Delta u_{m}(t)\right|_{2}^{2}+\frac{1}{4 \lambda}\left|u_{m}^{\prime}(t)\right|_{2}^{2}
\end{aligned}
$$

y

$$
\int_{0}^{t}|h(s)|_{2}\left|\Delta u_{m}(s)\right|_{2} d s \leq \eta \int_{0}^{t}\left|\Delta u_{m}(s)\right|_{2}^{2} d s+\frac{1}{4 \eta} \int_{0}^{t}|h(s)|_{2}^{2} d s,
$$

donde $0<\lambda<\frac{1}{2}$ constante.

Integrando (3.28), usando (3.29) - (3.31), y las estimativas (3.12) y (3.25), obtenemos

$$
\begin{aligned}
\left(\frac{1}{2}-\lambda\right)\left|\Delta u_{m}(t)\right|_{2}^{2}+ & \int_{0}^{t}(\mu(s)-2 \eta)\left|\Delta u_{m}(s)\right|_{2}^{2} d s \\
\leq & \frac{1}{2}\left|\Delta u_{0 m}\right|_{2}^{2}+\left|u_{1 m}\right|_{2}\left|\Delta u_{0 m}\right|_{2}+\frac{K_{1}}{4 \lambda} \\
& +\frac{1}{4 \eta} \int_{0}^{t}|h(s)|_{2}^{2} d s+K_{2} T \\
& +\frac{1}{4 \eta}\left|g_{0}\right|_{L^{1}}^{2} \int_{0}^{t}\left|\Delta u_{m}(s)\right|_{2}^{2} d s
\end{aligned}
$$


De (3.32), escogiendo $\lambda=\frac{1}{4}$ y $\eta=\frac{3}{8} m_{0}$, resulta

$$
\begin{aligned}
\frac{1}{4}\left|\Delta u_{m}(t)\right|_{2}^{2}+\frac{1}{4} m_{0} & \int_{0}^{t}\left|\Delta u_{m}(s)\right|_{2}^{2} d s \\
\leq & \frac{1}{2}\left|\Delta u_{0 m}\right|_{2}^{2}+\left|u_{1 m}\right|_{2}\left|\Delta u_{0 m}\right|_{2}+K_{1} \\
& +\frac{2}{3 m_{0}} \mid h \|_{L^{2}\left(0, T ; L^{2}(\Omega)\right)}^{2}+K_{2} T \\
& +\frac{2}{3 m_{0}}\left|g_{0}\right|_{L^{1}}^{2} \int_{0}^{t}\left|\Delta u_{m}(s)\right|_{2}^{2} d s
\end{aligned}
$$

De (3.33) por la Desigualdad de Gronwall, tenemos

$$
\left|\Delta u_{m}(t)\right|_{2}^{2}+\int_{0}^{t}\left|\Delta u_{m}(s)\right|_{2}^{2} d s \leq K_{3}, \forall t \in[0, T]
$$

donde $K_{3}$ es una constante positiva independiente de $m$.

Estimativa IV. Multiplicamos la desigualdad (3.9) por $\lambda, 0<\lambda \leq 1$, la desigualdad (3.28) por $\varepsilon, 0<\varepsilon \leq 1$ y sumando los dos resultados, se obtiene

$$
\begin{array}{r}
\frac{d}{d t} E_{3}(t)+(\lambda-\varepsilon)\left\|u_{m}^{\prime}(t)\right\|^{2}+\varepsilon(\mu(t)-\eta)\left|\Delta u_{m}(t)\right|_{2}^{2} \\
\leq|h(t)|_{2}\left[\lambda\left|u_{m}^{\prime}(t)\right|_{2}+\varepsilon\left|\Delta u_{m}(t)\right|_{2}\right] \\
+\frac{1}{2} \lambda\left|\mu^{\prime}(t)\right|\left\|u_{m}(t)\right\|^{2} \\
+\frac{\varepsilon}{4 \eta}\left|g_{0}\right|_{L^{1}}\left(g_{0} *\left|\Delta u_{m}\right|_{2}^{2}\right)(t)
\end{array}
$$

donde

$$
\begin{array}{r}
E_{3}(t):=\frac{1}{2} \lambda\left[\left|u_{m}^{\prime}(t)\right|_{2}^{2}+\mu_{0}(t)\left\|u_{m}(t)\right\|^{2}+\left(g_{0} \square \nabla u_{m}\right)(t)\right] \\
+\frac{1}{2} \varepsilon\left[\left|\Delta u_{m}(t)\right|_{2}^{2}-2\left(u_{m}^{\prime}(t), \Delta u_{m}(t)\right)\right]
\end{array}
$$

y aquí tomamos $0<\eta \leq \frac{1}{2}\left|g_{0}\right|_{L^{1}}$.

De (3.36), utilizando $\left|\varepsilon\left(u_{m}^{\prime}(t), \Delta u_{m}(t)\right)\right| \leq \frac{\varepsilon}{\lambda}\left|u_{m}^{\prime}(t)\right|_{2}^{2}+\frac{\varepsilon \lambda}{4}\left|\Delta u_{m}(t)\right|_{2}^{2}$, resulta

$$
\begin{array}{r}
E_{3}(t) \geq\left(\frac{1}{2} \lambda-\frac{\varepsilon}{\lambda}\right)\left|u_{m}^{\prime}(t)\right|_{2}^{2}+\varepsilon\left(\frac{1}{2}-\frac{\lambda}{4}\right)\left|\Delta u_{m}(t)\right|_{2}^{2} \\
+\frac{1}{2} \lambda \mu_{0}(t)\left\|u_{m}(t)\right\|^{2}+\frac{1}{2} \lambda\left(g_{0} \square \nabla u_{m}\right)(t) .
\end{array}
$$

También, se tiene

$$
\begin{array}{r}
E_{3}(t) \leq\left(\frac{1}{2} \lambda+\frac{\varepsilon}{\lambda}\right)\left|u_{m}^{\prime}(t)\right|_{2}^{2}+\varepsilon\left(\frac{1}{2}+\frac{\lambda}{4}\right)\left|\Delta u_{m}(t)\right|_{2}^{2} \\
+\frac{1}{2} \lambda \mu_{0}(t)\left\|u_{m}(t)\right\|^{2}+\frac{1}{2} \lambda\left(g_{0} \square \nabla u_{m}\right)(t) .
\end{array}
$$


De (3.37) y (3.38), escogiendo $\lambda=1$ y $\varepsilon=\frac{2}{5}$, resulta

$$
\begin{gathered}
E_{3}(t) \geq F(t), \forall t \in[0, T], \\
E_{3}(0) \leq E_{0}
\end{gathered}
$$

donde

$$
\begin{aligned}
& F(t):=\frac{1}{10}\left|u_{m}^{\prime}(t)\right|_{2}^{2}+\frac{1}{10}\left|\Delta u_{m}(t)\right|_{2}^{2}+\frac{1}{2} \mu_{0}(t)\left\|u_{m}(t)\right\|^{2}+\frac{1}{2}\left(g_{0} \square \nabla u_{m}\right)(t), \\
& E_{0}:=\frac{9}{10}\left|u_{1 m}\right|_{2}^{2}+\frac{3}{10}\left|\Delta u_{0 m}\right|_{2}^{2}+\frac{1}{2} \mu(0)\left\|u_{0 m}\right\|^{2} .
\end{aligned}
$$

De (3.35), se obtiene

$$
\begin{array}{r}
\frac{1}{2} \frac{d}{d t} E_{3}(t)+\frac{3}{10}\left\|u_{m}^{\prime}(t)\right\|^{2}+\frac{1}{5}\left(m_{0}-\eta\right)\left|\Delta u_{m}(t)\right|_{2}^{2} \\
\leq|h(t)|_{2}\left[E_{3}^{\frac{1}{2}}(t)+E_{3}^{\frac{1}{2}}(t)\right] \\
\cdot+\frac{1}{2} \frac{\left|\mu^{\prime}(t)\right|}{\mu_{0}(t)} E_{3}(t) \\
+\frac{1}{20 \eta}\left|g_{0}\right|_{L^{1}}\left(g_{0} *\left|\Delta u_{m}\right|_{2}^{2}\right)(t) .
\end{array}
$$

Integrando (3.41) y usando (3.29), obtenemos

$$
\begin{aligned}
& \frac{1}{2} E_{3}(t)+\frac{3}{10} \int_{0}^{t}\left\|u_{m}^{\prime}(s)\right\|^{2} d s \\
& +\frac{1}{5}\left(m_{0}-\eta-\frac{1}{4 \eta}\left|g_{0}\right|_{L^{1}}^{2}\right) \int_{0}^{t}\left|\Delta u_{m}(s)\right|_{2}^{2} d s \\
& \quad \leq \frac{1}{2} E_{3}(0)+2 \int_{0}^{t}|h(s)|_{2} E_{3}^{\frac{1}{2}}(s) d s+\frac{1}{2} \int_{0}^{t} \frac{\left|\mu^{\prime}(s)\right|}{\mu_{0}(s)} E_{3}(s) d s .
\end{aligned}
$$

De (3.42), escogiendo $\eta=\frac{1}{2}\left|g_{0}\right|_{L^{1}}$ y utilizando (3.39) - (3.40), resulta

$$
\begin{aligned}
\frac{1}{2} E_{2}(t) \leq \frac{1}{2} E_{0} & +2 \int_{0}^{t}|h(s)|_{2} E_{2}^{\frac{1}{2}}(s) d s \\
& +\frac{1}{2} \int_{0}^{t} \frac{\left|\mu^{\prime}(s)\right|}{\mu_{0}(s)} E_{2}(s) d s,
\end{aligned}
$$

donde

$$
\begin{aligned}
E_{2}(t): & =\frac{1}{10}\left|u_{m}^{\prime}(t)\right|_{2}^{2}+\frac{1}{10}\left|\Delta u_{m}(t)\right|_{2}^{2}+\frac{1}{2} \mu_{0}(t)\left\|u_{m}(t)\right\|^{2} \\
& +\frac{1}{2}\left(g_{0} \square \nabla u_{m}\right)(t)+\frac{3}{5} \int_{0}^{t}\left\|u_{m}^{\prime}(s)\right\|^{2} d s+\frac{2}{5} l_{0} \int_{0}^{t}\left|\Delta u_{m}(s)\right|_{2}^{2} d s .
\end{aligned}
$$

De (3.43), por la Desigualdad de Gronwall, resulta la estimativa

$$
E_{2}(t) \leq\left[E_{0}^{\frac{1}{2}}+2 \int_{0}^{t}|h(s)|_{2} d s\right]^{2} \exp \left(\int_{0}^{t} \frac{\left|\mu^{\prime}(s)\right|}{\mu_{0}(s)} d s\right), \forall t \in[0, T] .
$$


Pasaje al límite. Sean $j \geq m$ dos números naturales y consideremos $v_{m}=u_{j}-u_{m}$. Entonces (3.44) será de la forma

$$
E_{2}(t) \leq E_{0} \exp \left(\int_{0}^{t} \frac{\left|\mu^{\prime}(s)\right|}{\mu_{0}(s)} d s\right), \forall t \in[0, T] .
$$

Desde que $\left\{u_{0 m}\right\}$ y $\left\{u_{1 m}\right\}$ son sucesiones de Cauchy en $H_{0}^{1}(\Omega) \cap H^{2}(\Omega)$ y $L^{2}(\Omega)$, respectivamente, por (3.45) se obtienen

$$
\begin{gathered}
\left|\Delta v_{m}(t)\right|_{2}^{2} \rightarrow 0,\left\|v_{m}(t)\right\|^{2} \rightarrow 0, \quad \int_{0}^{t}\left|\Delta v_{m}(s)\right|_{2}^{2} d s \rightarrow 0, \\
\left|v_{m}^{\prime}(t)\right|_{2}^{2} \rightarrow 0 \text { y } \int_{0}^{t}\left\|v_{m}^{\prime}(s)\right\|^{2} d s \rightarrow 0,
\end{gathered}
$$

cuando $m \rightarrow \infty, \forall t \in[0, T]$.

Por las estimativas (3.12), (3.25) y (3.34), y las convergencias (3.46) y (3.47), obtenemos

$$
\begin{array}{lll}
u_{m} \rightarrow u & \text { fuerte en } & C\left([0, T] ; H_{0}^{1}(\Omega) \cap H^{2}(\Omega)\right), \\
u_{m}^{\prime} \rightarrow u^{\prime} & \text { fuerte en } & C\left([0, T] ; L^{2}(\Omega)\right), \\
u_{m}^{\prime} \rightarrow u^{\prime} & \text { fuerte en } & L^{2}\left(0, T ; H_{0}^{1}(\Omega)\right), \\
u_{m}^{\prime \prime} \rightarrow u^{\prime \prime} & \text { debil en } & L^{2}\left(0, T ; L^{2}(\Omega)\right) .
\end{array}
$$

Por (3.48) y de la ecuación en (3.3), por pasaje al límite, resulta

$$
u^{\prime \prime}-\mu(t) \Delta u+g_{0} * \Delta u-\Delta u^{\prime}=h(x, t) \text { en } L^{2}\left(0, T ; H^{-1}(\Omega)\right) .
$$

Los datos iníciales se verifican de modo estandar. La estimativa (3.2) se obtiene de manera similar que (3.44). La unicidad resulta de la estimativa (3.44). Esto concluye la demostración del Teorema 3.1 .

\section{EXISTENCIA LOCAL}

El objetivo principal del presente trabajo es discutir la existencia de soluciones del problema (1.1) - (1.6), usando argumentos del Teorema de Punto Fijo de Banach. Para esto emplearemos resultados del Teorema 3.1.

Definamos el siguiente espacio a dos parámetros, llamado Conjunto de Soluciones o Conjunto Admisible

$$
\begin{aligned}
G_{T_{0}, R_{0}}:=\left\{(u, v) \in X^{2} ; u^{\prime}, v^{\prime} \in C\left(\left[0, T_{0}\right] ; L^{2}(\Omega)\right) \cap L^{2}\left(0, T_{0} ; H_{0}^{1}(\Omega)\right)\right. \\
\quad e(u(t), v(t)) \leq R_{0}^{2}, t \in\left[0, T_{0}\right] \\
\left.\quad \operatorname{con}(u(0), v(0))=\left(u_{0}, v_{0}\right) \text { y }\left(u^{\prime}(0), v^{\prime}(0)\right)=\left(u_{1}, v_{1}\right)\right\},
\end{aligned}
$$

donde $X:=C\left(\left[0, T_{0}\right] ; H_{0}^{1}(\Omega) \cap H^{2}(\Omega)\right), e(u(t), v(t)):=\left|u^{\prime}(t)\right|_{2}^{2}+|\Delta u(t)|_{2}^{2}+\left|v^{\prime}(t)\right|_{2}^{2}+|\Delta v(t)|_{2}^{2}$, $T_{0}>0$ y $R_{0}>0$. Entonces $G_{T_{0}, R_{0}}$ es un espacio métrico completo con la distancia

$$
d(U, V):=\sup _{0 \leq t \leq T_{0}}\left[e\left(\left(u-u_{2}\right)(t),\left(v-v_{2}\right)(t)\right)\right]^{\frac{1}{2}},
$$

donde $U=(u, v), V=\left(u_{2}, v_{2}\right)$. 
Lema 4.1. Supongamos que la función $M$ satisface la hipótesis (H3). Si $(u, v) \in G_{T_{0}, R_{0}} y$ $\mu(t ; u):=M\left(\|u(t)\|^{2}\right)$, entonces $\mu(. ; u) \in C\left(\left[0, T_{0}\right]\right), \mu^{\prime}(. ; u) \in L^{\infty}\left(0, T_{0}\right)$ y se cumplen para cada $t, s \in\left[0, T_{0}\right]$ las siguientes desigualdades:

$$
\begin{gathered}
|\mu(t ; u)-\mu(s ; u)| \leq 2 M_{1} R_{0}^{2}|t-s| \\
|\mu(t ; u)-\mu(t ; v)| \leq 2 M_{1} B_{1}^{2} R_{0}|\Delta u(t)-\Delta v(t)|_{2}, \\
\left|\mu^{\prime}(t ; u)\right| \leq 2 M_{1} R_{0}^{2} \\
0<m_{0} \leq \mu(t ; u) \leq M_{0},
\end{gathered}
$$

donde $M_{0}:=\sup \left\{M(s) ; 0 \leq s \leq B_{1}^{2} R_{0}^{2}\right\}, B_{1}$ es la constante de la Desigualdad de SobolevPoincaré y $M_{1}:=\sup \left\{\left|M^{\prime}(s)\right| ; 0 \leq s \leq B_{1}^{2} R_{0}^{2}\right\}$.

\section{Demostración.}

Por el Teorema de Valor Medio, se tiene que

$$
|\mu(t ; u)-\mu(s ; u)|=\left|2 M^{\prime}\left(\|u(\xi)\|^{2}\right)\left(-\Delta u(\xi), u^{\prime}(\xi)\right)\right||t-s|
$$

y

$$
\begin{aligned}
|\mu(t ; u)-\mu(t ; v)| & =\left|M^{\prime}(\eta)\right|(\|u(t)\|+\|v(t)\|)|\|u(t)\|-\|v(t)\|| \\
& \leq\left|M^{\prime}(\eta)\right|(\|u(t)\|+\|v(t)\|)\|u(t)-v(t)\|,
\end{aligned}
$$

donde $\xi$ está entre $t$ y $s, \eta$ está entre $\|u(t)\|^{2}$ y $\|v(t)\|^{2}$. Por aplicación de la Desigualdad de Sobolev-Poincaré, a las relaciones anteriores se obtienen los resultados.

Lema 4.2. Supongamos que las funciones $f_{i}, i=1,2$, satisfacen la hipótesis (H4). Si $(u, v),\left(u_{2}, v_{2}\right) \in G_{T_{0}, R_{0}}$ y $h_{i}(t ; u, v):=f_{i}(u(t), v(t))$, entonces $h_{i} \in C\left(\left[0, T_{0}\right] ; L^{2}(\Omega)\right)$ y se verifican para cada $t, s \in\left[0, T_{0}\right]$ las siguientes desigualdades:

$$
\begin{aligned}
&\left|h_{i}(t ; u, v)-h_{i}(s ; u, v)\right|_{2} \leq 2 B_{\alpha \beta}\left[R_{0}^{\alpha}|\Delta u(t)-\Delta u(s)|_{2}\right. \\
&\left.+R_{0}^{\beta}|\Delta v(t)-\Delta v(s)|_{2}\right], \\
&\left|h_{i}(t ; u, v)-h_{i}\left(t ; u_{2}, v_{2}\right)\right|_{2} \leq 2 B_{\alpha \beta}\left[R_{0}^{\alpha}\left|\Delta u(t)-\Delta u_{2}(t)\right|_{2}\right. \\
&\left.+R_{0}^{\beta}\left|\Delta v(t)-\Delta v_{2}(t)\right|_{2}\right], \\
&\left|h_{i}(t ; u, v)\right|_{2} \leq B_{\alpha \beta}\left[R_{0}^{\alpha+1}+R_{0}^{\beta+1}\right],
\end{aligned}
$$

donde $B_{\alpha \beta}:=K \operatorname{máx}\left\{B_{1}^{2(\alpha+1)}, B_{1}^{2(\beta+1)}\right\}$ y $B_{1}$ es la constante de la Desigualdad de SobolevPoincaré. 
Por la hipótesis $(H 4)$, resulta

$$
\begin{aligned}
\left|h_{i}(t ; u, v)-h_{i}(s ; u, v)\right|_{2}= & \left|f_{i}(u(t), v(t))-f_{i}(u(s), v(s))\right|_{2} \\
\leq & K\left|\left(|u(t)|^{\alpha}+|u(s)|^{\alpha}\right)\right| u(t)-u(s) \mid \\
& +\left.\left(|v(t)|^{\beta}+|v(s)|^{\beta}\right)|v(t)-v(s)|\right|_{2} \\
\leq & K\left[\left|\left(|u(t)|^{\alpha}+|u(s)|^{\alpha}\right)\right| u(t)-\left.u(s)\right|_{2}\right. \\
& \left.+\left|\left(|v(t)|^{\beta}+|v(s)|^{\beta}\right)\right| v(t)-v(s)||_{2}\right] \\
\leq & K\left[\left(|u(t)|_{r \alpha}^{\alpha}+|u(s)|_{r \alpha}^{\alpha}\right)|u(t)-u(s)|_{q}\right. \\
& \left.+\left(|v(t)|_{r \beta}^{\beta}+|v(s)|_{r \beta}^{\beta}\right)|v(t)-v(s)|_{q}\right],
\end{aligned}
$$

donde $\frac{1}{r}+\frac{1}{q}=\frac{1}{2}$. Aplicando la Desigualdad de Sobolev-Poincaré, se obtiene

$$
\begin{aligned}
\left|h_{i}(t ; u, v)-h_{i}(s ; u, v)\right|_{2} \leq & B_{\alpha \beta}\left[\left(|\Delta u(t)|_{2}^{\alpha}+|\Delta u(s)|_{2}^{\alpha}\right)|\Delta u(t)-\Delta u(s)|_{2}\right. \\
& \left.+\left(|\Delta v(t)|_{2}^{\beta}+|\Delta v(s)|_{2}^{\beta}\right)|\Delta v(t)-\Delta v(s)|_{2}\right] \\
\leq & 2 B_{\alpha \beta}\left[R_{0}^{\alpha}|\Delta u(t)-\Delta u(s)|_{2}\right. \\
& \left.+R_{0}^{\beta}|\Delta v(t)-\Delta v(s)|_{2}\right] .
\end{aligned}
$$

Recurriendo nuevamente a la hipotesis $(H 4)$ y la Desigualdad de Sobolev-Poincaré, se obtiene

$$
\begin{aligned}
\left|h_{i}(t ; u, v)\right|_{2} & =\left|f_{i}(u(t), v(t))\right|_{2} \\
& \leq K\left[|u(t)|_{2(\alpha+1)}^{\alpha+1}+|v(t)|_{2(\beta+1)}^{\beta+1}\right] \\
& \leq K\left[B_{1}^{2(\alpha+1)}|\Delta u(t)|_{2}^{\alpha+1}+B_{1}^{2(\beta+1)}|\Delta v(t)|_{2}^{\beta+1}\right] \\
& \leq K\left[B_{1}^{2(\alpha+1)} R_{0}^{\alpha+1}+B_{1}^{2(\beta+1)} R_{0}^{(\beta+1)}\right] \\
& \leq B_{\alpha \beta}\left[R_{0}^{\alpha+1}+R_{0}^{(\beta+1)}\right] .
\end{aligned}
$$

Observar, que en la aplicación de la Desigualdad de Sobolev-Poincaré, se han utilizado las condiones de $\alpha$ y $\beta$. Esto concluye la demostración.

Teorema 4.3 (Existencia Local). Supongamos que las funciones $g_{i}, M$ y $f_{i}$ satisfacen las hipótesis (H2), (H3) y (H4), respectivamente, $u_{0}, v_{0} \in H_{0}^{1}(\Omega) \cap H^{2}(\Omega)$ y $u_{1}, v_{1} \in L^{2}(\Omega)$. Entonces existe $T_{0}>0$, de tal modo que el problema $(1.1)$ - (1.6) admite solución única $(u, v)$ sobre $\left[0, T_{0}\right]$ tal que

$$
\begin{aligned}
& u, v \in C\left(\left[0, T_{0}\right] ; H_{0}^{1}(\Omega) \cap H^{2}(\Omega)\right), \\
& u^{\prime}, v^{\prime} \in C\left(\left[0, T_{0}\right] ; L^{2}(\Omega)\right) \cap L^{2}\left(0, T_{0} ; H_{0}^{1}(\Omega)\right), \\
& u^{\prime \prime}, v^{\prime \prime} \in L^{2}\left(0, T_{0} ; L^{2}(\Omega)\right) .
\end{aligned}
$$

Demostración. Procedemos en dos etapas.

Existencia de Soluciones. Para cada $\bar{U}=(\bar{u}, \bar{v}) \in G_{T_{0}, R_{0}}$, consideremos el siguiente sistema lineal

$$
\begin{array}{cl}
u^{\prime \prime}-\mu(t ; \bar{u}) \Delta u+g_{1} * \Delta u-\Delta u^{\prime}=h_{1}(t ; \bar{u}, \bar{v}) & \text { en } \Omega \times] 0, T_{0}[, \\
v^{\prime \prime}-\mu(t ; \bar{v}) \Delta v+g_{2} * \Delta v-\Delta v^{\prime}=h_{2}(t ; \bar{u}, \bar{v}) & \text { en } \Omega \times] 0, T_{0}[,
\end{array}
$$


con condiciones iníciales

$$
\begin{aligned}
& u(x, 0)=u_{0}(x), u^{\prime}(x, 0)=u_{1}(x), \text { en } \Omega, \\
& v(x, 0)=v_{0}(x), v^{\prime}(x, 0)=v_{1}(x), \text { en } \Omega,
\end{aligned}
$$

y condiciones de frontera

$$
\begin{aligned}
& u(x, t)=0, \text { en } \partial \Omega \times] 0, T_{0}[, \\
& v(x, t)=0, \text { en } \partial \Omega \times] 0, T_{0}[,
\end{aligned}
$$

donde $T_{0}>0$ y $R_{0}>0$ serán obtenidos posteriormente, $\mu(t ; \bar{w}):=M\left(\|\bar{w}(t)\|^{2}\right), h_{1}(t ; \bar{u}, \bar{v}):=$ $f_{1}(\bar{u}(t), \bar{v}(t))$ y $h_{2}(t ; \bar{u}, \bar{v}):=f_{2}(\bar{u}(t), \bar{v}(t))$. Por los Lemas 4.1 y 4.2 , las funciones $\mu(. ; \bar{u})$, $\mu(. ; \bar{v}), h_{1}(. ; \bar{u}, \bar{v})$ y $h_{2}(. ; \bar{u}, \bar{v})$, satisfacen las hipótesis del Teorema 3.1, entonces existe solución única, $U=(u, v)$ sobre $\left[0, T_{0}\right]$ del problema $(4.2)-(4.7)$ tal que

$$
\begin{aligned}
& u, v \in C\left(\left[0, T_{0}\right] ; H_{0}^{1}(\Omega) \cap H^{2}(\Omega)\right), \\
& u^{\prime}, v^{\prime} \in C\left(\left[0, T_{0}\right] ; L^{2}(\Omega)\right) \cap L^{2}\left(0, T_{0} ; H_{0}^{1}(\Omega)\right), \\
& u^{\prime \prime}, v^{\prime \prime} \in L^{2}\left(0, T_{0} ; L^{2}(\Omega)\right) .
\end{aligned}
$$

Además, la solución $U=(u, v)$ verifica para cada $t \in\left[0, T_{0}\right]$, las siguientes estimativas

$$
E(t ; u) \leq\left[E_{(0 ; u)}^{\frac{1}{2}}+2 \int_{0}^{t}\left|h_{1}(s ; \bar{u}, \bar{v})\right|_{2} d s\right]^{2} \exp \left(\int_{0}^{t} \frac{\left|\mu^{\prime}(s ; \bar{u})\right|}{\mu_{1}(s ; \bar{u})} d s\right)
$$

y

$$
E(t ; v) \leq\left[E_{(0 ; v)}^{\frac{1}{2}}+2 \int_{0}^{t}\left|h_{2}(s ; \bar{u}, \bar{v})\right|_{2} d s\right]^{2} \exp \left(\int_{0}^{t} \frac{\left|\mu^{\prime}(s ; \bar{v})\right|}{\mu_{2}(s ; \bar{v})} d s\right),
$$

donde

$$
\begin{aligned}
& E(t ; w):=\frac{1}{10}\left|w^{\prime}(t)\right|_{2}^{2}+\frac{1}{10}|\Delta w(t)|_{2}^{2}+\frac{1}{2} \mu_{i}(t ; \bar{w})\|w(t)\|^{2}, \\
& \mu_{i}(t ; \bar{w}):=\mu(t ; \bar{w})-\int_{0}^{t} g_{i}(s) d s \text { para } i=1,2, \\
& E_{(0 ; w)}:=\frac{9}{10}\left|w_{1}\right|_{2}^{2}+\frac{3}{10}\left|\Delta w_{0}\right|_{2}^{2}+\frac{1}{2} \mu(0)\left\|w_{0}\right\|^{2} .
\end{aligned}
$$

El siguiente paso será mostrar que $U=(u, v) \in G_{T_{0}, R_{0}}$. Por (4.9), (4.10) y los Lemas 4.1 y 4.2 , obtenemos

$$
\begin{array}{r}
\left|u^{\prime}(t)\right|_{2}^{2}+|\Delta u(t)|_{2}^{2} \leq 10\left[E_{(0 ; u)}^{\frac{1}{2}}+2 B_{\alpha \beta}\left(R_{0}^{\alpha+1}+R_{0}^{\beta+1}\right) T_{0}\right]^{2} \\
\exp \left(\frac{2 M_{1} R_{0}^{2} T_{0}}{l}\right)
\end{array}
$$

y

$$
\begin{array}{r}
\left|v^{\prime}(t)\right|_{2}^{2}+|\Delta v(t)|_{2}^{2} \leq 10\left[E_{(0 ; v)}^{\frac{1}{2}}+2 B_{\alpha \beta}\left(R_{0}^{\alpha+1}+R_{0}^{\beta+1}\right) T_{0}\right]^{2} \\
\exp \left(\frac{2 M_{1} R_{0}^{2} T_{0}}{l}\right),
\end{array}
$$

donde $l:=\operatorname{mín}\left\{l_{1}, l_{2}\right\}$. Sumando (4.11) y (4.12), resulta

$$
\begin{array}{r}
e(u(t), v(t)) \leq 10 \exp \left(\frac{2 M_{1} R_{0}^{2} T_{0}}{l}\right) \\
{\left[\left(E_{(0 ; u)}^{\frac{1}{2}}+2 B_{\alpha \beta}\left(R_{0}^{\alpha+1}+R_{0}^{\beta+1}\right) T_{0}\right)^{2}\right.} \\
\left.+\left(E_{(0 ; v)}^{\frac{1}{2}}+2 B_{\alpha \beta}\left(R_{0}^{\alpha+1}+R_{0}^{\beta+1}\right) T_{0}\right)^{2}\right] .
\end{array}
$$


Escogemos la constante $R_{0}>0$ que satisfaga la relación

$$
R_{0} \geq 10\left[\left(E_{(0 ; u)}^{\frac{1}{2}}+2\right)^{2}+\left(E_{(0 ; v)}^{\frac{1}{2}}+2\right)^{2}\right]^{\frac{1}{2}}
$$

y consideremos $T_{0}>0$ que satisfaga las relaciones

$$
B_{\alpha \beta}\left(R_{0}^{\alpha+1}+R_{0}^{\beta+1}\right) T_{0} \leq 1 \text { y } \exp \left(\frac{2 M_{1} R_{0}^{2} T_{0}}{l}\right) \leq 10 .
$$

Entonces de (4.13) utilizando (4.14) y (4.15), resulta

$$
e(u(t), v(t)) \leq R_{0}^{2}, \forall t \in\left[0, T_{0}\right] .
$$

Por (4.16), (4.8) y como $(u, v)$ es solución de (4.2) - (4.7), se tiene que $(u, v) \in G_{T_{0}, R_{0}}$.

Por el resultado antes obtenido, tieene sentido definir la aplicación no lineal $S$ como sigue:

$$
\begin{aligned}
& S: G_{T_{0}, R_{0}} \longrightarrow G_{T_{0}, R_{0}} \quad \text {. } \\
& (\bar{u}, \bar{v}) \quad \longmapsto S(\bar{u}, \bar{v})=(u, v)
\end{aligned}
$$

donde $(u, v)$ es la solución del problema $(4.2)-(4.7)$. Esto significa que $S\left(G_{T_{0}, R_{0}}\right) \subset G_{T_{0}, R_{0}}$.

Ahora probaremos que $S$ es una contracción estricta con respecto a la distancia (4.1), es decir, se cumple para cada $\bar{U}=(\bar{u}, \bar{v}), \bar{V}=\left(\bar{u}_{2}, \bar{v}_{2}\right) \in G_{T_{0}, R_{0}}$ la desigualdad

$$
d(S(\bar{U}), S(\bar{V})) \leq \delta d(\bar{U}, \bar{V})
$$

para un fijo $\delta, 0<\delta<1$.

Sean $\bar{U}=(\bar{u}, \bar{v}), \bar{V}=\left(\bar{u}_{2}, \bar{v}_{2}\right) \in G_{T_{0}, R_{0}}$, entonces $U=S(\bar{U})$ y $V=S(\bar{V})$ son soluciones del problema (4.2)-(4.7), donde $U=(u, v)$ y $V=\left(u_{2}, v_{2}\right)$. Haciendo, $\left.Q:=\Omega \times\right] 0, T_{0}[, \Sigma:=\partial \Omega \times] 0, T_{0}[$ , $w_{1}=u-u_{2}$ y $w_{2}=v-v_{2}$, entonces $W=\left(w_{1}, w_{2}\right)$ satisface el problema lineal

$$
\begin{array}{ll}
w_{1}^{\prime \prime}-\mu(t ; \bar{u}) \Delta w_{1}+g_{1} * \Delta w_{1}-\Delta w_{1}^{\prime}=h_{1}(t ; \bar{U}, \bar{V}) & \text { en } Q, \\
w_{2}^{\prime \prime}-\mu(t ; \bar{v}) \Delta w_{2}+g_{2} * \Delta w_{2}-\Delta w_{2}^{\prime}=h_{2}(t ; \bar{U}, \bar{V}) & \text { en } Q, \\
w_{1}=0, w_{2}=0 & \text { en } \Sigma, \\
w_{1}(0)=0, w_{1}^{\prime}(0)=0, w_{2}(0)=0, w_{2}^{\prime}(0)=0 & \text { en } \Omega,
\end{array}
$$

donde

$$
\begin{aligned}
& \mu(t ; z):=M\left(\|z(t)\|^{2}\right), \\
& h_{1}(t ; \bar{U}, \bar{V}):=\left[\mu(t ; \bar{u})-\mu\left(t ; \bar{u}_{2}\right)\right] \Delta u_{2}(t)+f_{1}(\bar{U}(t))-f_{1}(\bar{V}(t)), \\
& h_{2}(t ; \bar{U}, \bar{V}):=\left[\mu(t ; \bar{v})-\mu\left(t ; \bar{v}_{2}\right)\right] \Delta v_{2}(t)+f_{2}(\bar{U}(t))-f_{2}(\bar{V}(t)) .
\end{aligned}
$$

Por los Lemas 4.1 y 4.2 , obtenemos

$$
\left|h_{i}(t ; \bar{U}, \bar{V})\right|_{2} \leq 2 B_{R_{0}} e^{\frac{1}{2}}\left(\left(\bar{u}-\bar{u}_{2}\right)(t),\left(\bar{v}-\bar{v}_{2}\right)(t)\right),
$$

donde $B_{R_{0}}:=M_{1} B_{1}^{2} R_{0}^{2}+B_{\alpha \beta}\left(R_{0}^{\alpha}+R_{0}^{\beta}\right)$. Del Lema 4.1, (4.18) y Teorema 3.1, existe una única solución $W=\left(w_{1}, w_{2}\right)$ sobre $\left[0, T_{0}\right]$ del problema (4.17) y se verifica para cada $t \in\left[0, T_{0}\right]$ las estimativas

$$
E\left(t ; w_{1}\right) \leq\left[2 \int_{0}^{t}\left|h_{1}(s ; \bar{U}, \bar{V})\right|_{2} d s\right]^{2} \exp \left(\int_{0}^{t} \frac{\left|\mu^{\prime}(s ; \bar{u})\right|}{\mu_{1}(s ; \bar{u})} d s\right)
$$


y

$$
E\left(t ; w_{2}\right) \leq\left[2 \int_{0}^{t}\left|h_{2}(s ; \bar{U}, \bar{V})\right|_{2} d s\right]^{2} \exp \left(\int_{0}^{t} \frac{\left|\mu^{\prime}(s ; \bar{v})\right|}{\mu_{2}(s ; \bar{v})} d s\right)
$$

donde

$$
\begin{aligned}
& E\left(t ; w_{1}\right):=\frac{1}{10}\left|w_{1}^{\prime}(t)\right|_{2}^{2}+\frac{1}{10}\left|\Delta w_{1}(t)\right|_{2}^{2}+\frac{1}{2} \mu_{i}(t ; \bar{u})\left\|w_{1}(t)\right\|^{2}, \\
& E\left(t ; w_{2}\right):=\frac{1}{10}\left|w_{2}^{\prime}(t)\right|_{2}^{2}+\frac{1}{10}\left|\Delta w_{2}(t)\right|_{2}^{2}+\frac{1}{2} \mu_{i}(t ; \bar{v})\left\|w_{2}(t)\right\|^{2}, \\
& \mu_{i}(t ; \bar{w}):=\mu(t ; \bar{w})-\int_{0}^{t} g_{i}(s) d s \text { para } i=1,2 .
\end{aligned}
$$

Sumando (4.19) y (4.20), utilizando (4.15) y (4.18), se obtiene

$$
e\left(w_{1}(t), w_{2}(t)\right) \leq 1600 B_{R_{0}}^{2} T_{0}^{2} e\left(\left(\bar{u}-\bar{u}_{2}\right)(t),\left(\bar{v}-\bar{v}_{2}\right)(t)\right) .
$$

Aplicando supremo en (4.21), resulta

$$
d(S(\bar{U}), S(\bar{V})) \leq 1600 B_{R_{0}}^{2} T_{0}^{2} d(\bar{U}, \bar{V}) .
$$

Además de las relaciones de (4.15), y tomando un $T_{0}>0$ que satisfaga la relación

$$
\delta:=1600 B_{R_{0}}^{2} T_{0}^{2}<1
$$

se tiene de (4.22) que $S$ es una contracción estricta.

Aplicando el teorema de punto fijo de Banach, existe un único $(u, v) \in G_{T_{0}, R_{0}}$ tal que $S(u, v)=(u, v)$. Así, hemos obtenido la existencia de la solución local del problema (1.1) - (1.6)

Unicidad de Soluciones. Sea $U=(u, v)$ la solución obtenida en la prueba de existencia, la cual pertenece a $G_{T_{0}, R_{0}}$. Consideremos otra solución $V=\left(u_{2}, v_{2}\right)$ del problema (1.1) - (1.6) tal que

$$
\begin{aligned}
& u_{2}, v_{2} \in C\left(\left[0, T_{1}\right] ; H_{0}^{1}(\Omega) \cap H^{2}(\Omega)\right), \\
& u_{2}^{\prime}, v_{2}^{\prime} \in C\left(\left[0, T_{1}\right] ; L^{2}(\Omega)\right) \cap L^{2}\left(0, T_{1} ; H_{0}^{1}(\Omega)\right), \\
& u_{2}^{\prime \prime}, v_{2}^{\prime \prime} \in L^{2}\left(0, T_{1} ; L^{2}(\Omega)\right),
\end{aligned}
$$

con $0<T_{1} \leq T_{0}$. Sean $w_{1}=u-u_{2}$ y $w_{2}=v-v_{2}$. Entonces el par de funciones $\left(w_{1}, w_{2}\right)$ satisfacen el siguiente problema lineal

$$
\begin{array}{ll}
w_{1}^{\prime \prime}-\mu(t ; u) \Delta w_{1}+g_{1} * \Delta w_{1}-\Delta w_{1}^{\prime}=h_{1}(t ; U, V) & \text { en } Q, \\
w_{2}^{\prime \prime}-\mu(t ; v) \Delta w_{2}+g_{2} * \Delta w_{2}-\Delta w_{2}^{\prime}=h_{2}(t ; U, V) & \text { en } Q, \\
w_{1}=0, w_{2}=0 & \text { en } \Sigma, \\
w_{1}(0)=0, w_{1}^{\prime}(0)=0, w_{2}(0)=0, w_{2}^{\prime}(0)=0 & \text { en } \Omega,
\end{array}
$$

donde

$$
\begin{aligned}
& \mu(t ; z):=M\left(\|z(t)\|^{2}\right), \\
& h_{1}(t ; U, V):=\left[\mu(t ; u)-\mu\left(t ; u_{2}\right)\right] \Delta u_{2}(t)+f_{1}(U(t))-f_{1}(V(t)), \\
& h_{2}(t ; U, V):=\left[\mu(t ; v)-\mu\left(t ; v_{2}\right)\right] \Delta v_{2}(t)+f_{2}(U(t))-f_{2}(V(t)) .
\end{aligned}
$$

Por (4.23), existe una constante $C_{0}>0$ tal que

$$
\left|\Delta u_{2}(t)\right|_{2},\left|\Delta v_{2}(t)\right|_{2} \leq C_{0}, \forall t \in\left[0, T_{1}\right] .
$$


Por el Teorema de Valor Medio, Desigualdad de Sobolev-Poincaré y (4.25), se obtienen

$$
\begin{array}{r}
\left|h_{1}(t ; U, V)\right|_{2} \leq M_{2} C_{0} B_{1}^{2}\left(R_{0}+C_{0}\right)\left|\Delta w_{1}(t)\right|_{2} \\
+K\left[B_{1}^{2(\alpha+1)}\left(R_{0}^{\alpha}+C_{0}^{\alpha}\right)\left|\Delta w_{1}(t)\right|_{2}\right. \\
\left.+B_{1}^{2(\beta+1)}\left(R_{0}^{\beta}+C_{0}^{\beta}\right)\left|\Delta w_{2}(t)\right|_{2}\right] \\
\leq \frac{K_{0}}{2} e^{\frac{1}{2}}\left(w_{1}(t), w_{2}(t)\right)
\end{array}
$$

y

$$
\begin{array}{r}
\left|h_{2}(t ; U, V)\right|_{2} \leq M_{2} C_{0} B_{1}^{2}\left(R_{0}+C_{0}\right)\left|\Delta w_{2}(t)\right|_{2} \\
+K\left[B_{1}^{2(\alpha+1)}\left(R_{0}^{\alpha}+C_{0}^{\alpha}\right)\left|\Delta w_{1}(t)\right|_{2}\right. \\
\left.+B_{1}^{2(\beta+1)}\left(R_{0}^{\beta}+C_{0}^{\beta}\right)\left|\Delta w_{2}(t)\right|_{2}\right] \\
\leq \frac{K_{0}}{2} e^{\frac{1}{2}}\left(w_{1}(t), w_{2}(t)\right)
\end{array}
$$

donde $M_{2}:=\sup \left\{\left|M^{\prime}(s)\right| ; 0 \leq s \leq B_{1}^{2}\right.$ máx $\left.\left\{R_{0}^{2}, C_{0}^{2}\right\}\right\}$ y $K_{0}$ es una constante positiva.

Del Lema 4.1, (4.15) y Teorema 3.1, existe una única solución $\left(w_{1}, w_{2}\right)$ sobre $\left[0, T_{1}\right]$ del problema (4.24) y se verifican las estimativas

$$
E\left(t ; w_{1}\right) \leq\left[2 \int_{0}^{t}\left|h_{1}(s ; U, V)\right|_{2} d s\right]^{2} \exp \left(\int_{0}^{t} \frac{\left|\mu^{\prime}(s ; u)\right|}{\mu_{1}(s ; u)} d s\right)
$$

y

$$
E\left(t ; w_{2}\right) \leq\left[2 \int_{0}^{t}\left|h_{2}(s ; U, V)\right|_{2} d s\right]^{2} \exp \left(\int_{0}^{t} \frac{\left|\mu^{\prime}(s ; v)\right|}{\mu_{2}(s ; v)} d s\right) .
$$

Sumando (4.28) y (4.29), y empleando (4.26) y (4.27), resulta

$$
e^{\frac{1}{2}}\left(w_{1}(t), w_{2}(t)\right) \leq K_{4} \int_{0}^{t} e^{\frac{1}{2}}\left(w_{1}(s), w_{2}(s)\right) d s, \forall t \in\left[0, T_{1}\right]
$$

donde $K_{4}:=4 K_{0} \exp \left(\frac{4 M_{1} R_{0}^{2} T_{0}}{l}\right)$ y $l:=\min \left\{l_{1}, l_{2}\right\}$.

De (4.30) y la Desigualdad de Gronwall, se tiene que $e^{\frac{1}{2}}\left(w_{1}(t), w_{2}(t)\right)=0, \forall t \in\left[0 . T_{1}\right]$, $0<T_{1} \leq T_{0}$. Esto implica que $w_{1}(t)=w_{2}(t)=0, \forall t \in\left[0, T_{1}\right]$. Con esto hemos probado la unicidad y por tanto finalizado la demostración del Teorema 4.3.

Corolario 4.4. Supongamos que las funciones $g_{i}, M$ y $f_{i}$ satisfacen las hipótesis (H2), (H3) y (H4), respectivamente, $u_{0}, v_{0} \in H_{0}^{1}(\Omega) \cap H^{2}(\Omega)$ y $u_{1}, v_{1} \in L^{2}(\Omega)$. Entonces existe un único intervalo $\left[0, T_{\operatorname{máx}}\left[\right.\right.$ con $0<T_{\text {máx }} \leq \infty$ y el problema $(1.1)-(1.6)$ admite solución única $(u, v)$ sobre $\left[0, T_{\operatorname{máx}}[\right.$ tal que

$$
\begin{aligned}
& u, v \in C\left(\left[0, T_{\text {máx }}\left[; H_{0}^{1}(\Omega) \cap H^{2}(\Omega)\right),\right.\right. \\
& u^{\prime}, v^{\prime} \in C\left(\left[0, T_{\text {máx }}\left[; L^{2}(\Omega)\right) \cap L^{2}\left(0, T_{\text {máx }} ; H_{0}^{1}(\Omega)\right),\right.\right. \\
& u^{\prime \prime}, v^{\prime \prime} \in L^{2}\left(0, T_{\text {máx }} ; L^{2}(\Omega)\right) .
\end{aligned}
$$

Demostración. Similar a la demostración del Corolario 4.4 [9]. 


\section{REFERENCIAS BIBLIOGRÁFICAS}

[1] Andrade D. and Mognon A., Global solutions for a system of Klein-Gordon equations with memory, Bol. Soc. Paran. Mat. 21 1/2 (2003).

[2] Brézis H., Análisis funcional, Alianza Editorial, Madrid, (1984).

[3] Coddington E. A. and Levinson N., Theory of ordinary differential equations, McGraw-Hill, New York, (1955).

[4] Kirchhoff G., Vorlesungen über mechanik, Leipzig, Teubner, (1883).

[5] Li, M.-R. and Tsai L.-Y., On a system of nonlinear wave equation, Taiwanese Journal of Mathematic Vol.7, No. 4, pp. 557-573, December (2003).

[6] Milla Miranda, M. and Medeiros, L. A., On the existence of global solutions of a coupled nonlinear Klein-Gordon equation, Funkcialaj Ekvacioj, 30(1987) 147-161.

[7] Quispe Méndez, T., Singularidad en tiempo finito para un sistema de Kirchhoff, Tesis de Maestria, Fac. CC. MM. de la UNMSM, Diciembre (1998).

[8] Quispe Méndez, T., Solución local y singularidad para un sistema de Kirchhoff no lineal, PESQUIMAT Revista de la Fac. CC. MM. de la Universidad Nacional Mayor de San Marcos Vol. XIII, No.2, pp 45-62, LIMA-PERÚ. Diciembre (2005).

[9] Quispe Méndez, T., Solución local de una ecuación de Kirchhoff no lineal viscoelástica con término disipativo, PESQUIMAT Revista de la Fac. CC. MM. de la Universidad Nacional Mayor de San Marcos Vol. X, No.1, pp 11-32, LIMA-PERÚ. Agosto (2007).

[10] Santos, M. L., Decay rates for solutions of a system of wave equations with memory, Electronic Journal of Differential Equations, Vol. 2002 (2002), No. 38, pp.1-17.

[11] Segal, I., Nonlinear partial differential equations in quantum fiels theory, Proc. Symp. Appl. Math. A.M.S., 17, 210-226 (1965).

[12] Wu S.-T., Blow-up of solutions for an integro-differential equation with a nonlinear source, Electronic Journal of Differential Equations, Vol. 2006 (2006), No. 45, pp.1-9.

[13] Wu S.-T. and Tsai L.-Y., On global existence and blow-up of solutions for an integrodifferential equation with strong damping, Taiwanese Journal of Mathematic Vol.10, No. 4, pp. 979-1014, June (2006).

[14] Wu S.-T. and Tsai L.-Y., On system of nonlinear wave equation of Kirchhoff type with a strong dissipation, Tamkang Journal of Mathematic Vol.38, No. 1, pp. 1.20, Spring (2007).

[15] Zeidler E., Nonlinear functional analysis and its applications I: Fixed-point theorems, Springer-Verlag, New York, (1986). 